\title{
Germanica
}

\section{Das neusachliche junge Mädchen zwischen Uznach und dem Oktoberfest}

De Sternheim à Horwath: la nouvelle jeune fille "objectiviste "

\section{Eva Philippoff}

\section{OpenEdition}

\section{Journals}

Édition électronique

URL : http://journals.openedition.org/germanica/2386

DOI : $10.4000 /$ germanica.2386

ISSN : 2107-0784

\section{Éditeur}

Université de Lille

\section{Édition imprimée}

Date de publication : 31 décembre 1991

Pagination : 105-121

ISSN : 0984-2632

\section{Référence électronique}

Eva Philippoff, «Das neusachliche junge Mädchen zwischen Uznach und dem Oktoberfest », Germanica [Online], 9 | 1991, Online erschienen am: 15 Juli 2014, abgerufen am 06 Oktober 2020. URL http://journals.openedition.org/germanica/2386 ; DOI : https://doi.org/10.4000/germanica.2386

Ce document a été généré automatiquement le 6 octobre 2020.

(c) Tous droits réservés 


\title{
Das neusachliche junge Mädchen zwischen Uznach und dem Oktoberfest
}

\author{
De Sternheim à Horwath: la nouvelle jeune fille "objectiviste »
}

\section{Eva Philippoff}

Wir Modernen haben gelernt, auch im Weibe den Menschen zu achten

1 Zwischen Uznach - Die Schule von Uznach oder Neue Sachlichen von Carl Sternheim ${ }^{2}$ und dem Oktoberfest - bekanntlich der Schauplatz von Horvàths Komödie Kasimir und Karoline $^{3}$ - liegen sechs Jahre, die Jahre von 1926 bis 1932. Diese sechs Jahre sollen hier aber nicht evolutiv verstanden sein, sondern symbolisch für den Kristallisationsraum unserer Betrachtungen über das «neue junge Mädchen», das Mädchen einer Zeit, die zugleich Nachkriegs - Krisen- und Vorkriegszeit war.

2 Angesichts der Tatsache, daß Sternheim eine Generation älter war als Horvàth ${ }^{4}$ und zum Thema schon vor ihm Aussagen bereit hatte, ziehen wir bei diesem Autor schon frühere Werke heran; bei beiden Autoren wird uns jedoch eine Zeitspanne von ungefähr zehn Jahren interessieren: 1920 - Der entfesselte Zeitgenosse - bis 1931 - Aut Caesar, aut nihil für Sternheim; 1926 - Zur schönen Aussicht - bis 1936 - Figaro läßt sich scheiden für Horvàth. Dazwischen liegen, um 1930 geballt, die so aufschlußreichen Romane von Marieluise Fleißer: Mehlreisende Frieda Geyer (1931), Irmgard Keun: Gilgi, eine von uns (1931) und Das kunstseidene Mädchen (1932), Erich Kästner: Fabian (1931) und Hans Fallada: Kleiner Mann, was nun? (1932).

3 Es ist natürlich schwierig, vielleicht sogar unmöglich, bei so verschiedenen Ansätzen und Standpunkten $\mathrm{zu}$ einer allgemeinen Synthese zu gelangen, große Unterschiede birgt schon die Tatsache, ob es sich um einen männlichen oder weiblichen Autor handelt. Es ist kein Zufall, daß die beiden Autorinnen, Fleißer und Keun, wie Livia Z. Wittmann in ihrem Artikel Der Stein des Anstoßes ${ }^{5}$ sehr richtig beanstandet, schnell in Vergessenheit gebracht wurden. Ihre Schlüsse unterscheiden sich wesentlich von 
denen der männlichen Kollegen: Im Gegensatz zu diesen wird bei Keun und Fleißer kein affektives und sentimentales Hintertürchen geöffnet! Doch davon später.

Wie sieht nun dieses neue junge Mädchen, Produkt der Kriegs und der Nachkriegszeit bei unseren Autoren aus? Als erster von ihnen nimmt es Sternheim ausdrücklich aufs Korn in seiner Komödie Der entfesselte Zeitgenosse. Die Heldin, Klara Cassati ist eine selbstbewußte junge Frau, mannigfach gebildet und ausgebildet - sie ist Erbin eines Riesenvermögens ${ }^{6}$ - und steht vor der Notwendigkeit, einen Gatten zu wählen, was ihr dank ihrer Scharfsichtigkeit und dank der Durchschnittlichkeit ihrer Bewerber nicht leicht fällt. Klara ist "gefrorene Vernunft, letzter Zeitgeist»", und "fast leibliche(r) Ekel» vor «Schwärmerei, Romantik» ${ }^{8}$, zeichnet sie aus. Sie besitzt «eines Philosophiestudenten Bildung, weiß nach vielen Seiten Theoretisches»" schätzt als «heutiges Gesetz: Ökonomie und ist begeisterter Zeitgenosse» ${ }^{10}$.

Das Glück, Millionenerbin zu sein und sich gelassen nach allen Seiten bilden zu können, um sich dann eingehend der Wahl eines Lebensgenossen zu widmen, zeichnet nur Sternheims Heldinnen aus. Die anderen Autoren, sozial nicht so hoch angesiedelt, liefern uns das Bild eines von den Härten des Daseins nicht verschonten jungen Mädchens: Mehlreisende Frieda Geyer z.B. trägt einen langen Herrenmantel, flache Schuhe, kurzes Haar und fährt aus Berufszwecken eine alte Knarre. In ihrem Beruf als Vertreterin muß sie sich gegen die Konkurrenz der Männer durchsetzen, noch dazu, da sie für die Erziehung einer jüngeren Schwester verantwortlich ist. «Man muß seinem Vordermann scharf auf die Hacken steigen, sonst wird man an die Peripherie gedrängt, wo man verhungert» ${ }^{11}$, ist gezwungenermaßen ihre Devise, obwohl sie manchmal lieber «abseits von der wilden Jagd gehen (möchte) und Gott danken für die Beere des Waldes, den Sauerampfer im Grase, den Schrei des Vogels um den Kirchturm und seinen blauen Himmel $»^{12}$.

6 Auch Gilgi, bzw. Gisela Kron, ist entschlossen, ihren Weg dank der eigenen Kraft zu gehen. Sie betreibt neben der Büroarbeit das Studium der Fremdsprachen, Gymnastik am Morgen und Brustschwimmen am Wochenende; auch sie haßt das Wort Sehnsucht, denn sie «will weiter, will selbständig und unabhängig sein»" ${ }^{13}$. Das bescheidene Ziel ihrer Hoffnung ist eine eigene Wohnung, vielleicht sogar einmal ein eigenes Modengeschäft.

7 Sachlichkeit, Zielstrebigkeit, Wunsch nach Unabhängigkeit - das zeichnet demnach das neue junge Mädchen aus. In seinem Stück Die Schule von Uznach oder Neue Sachlichkeit errichtet Sternheim diesem jungen Mädchen ein wahres Monument an Beredsamkeit. Nachdem es in den fünf Jahren seit Kriegsende mit dem Alten radikal aufgeräumt habe, «Gaurisankare europäischen Schutts in Brüche» ${ }^{14}$, «Schützengräben der Familie und ranziger Gesellschaft in Europa» $»^{15}$ gestürmt, und sich «keß in die Lebensschlacht» ${ }^{16}$ geworfen habe, sei es nun dabei, dank eurhythmischer Ballungen und Schwingungen, sich den «belämmerten Verhältnissen» ${ }^{17}$ der Zeit mutig zu stellen, das Leben zu reiten, und sich ohne Vorurteil den Anregungen des Tages offenzuhalten. «Stets mußt du für die höchste Daseinsform, die Ballung, bereit sein. Statt Einzeltöne wirst du Ballungsakkorde im All begreifen ${ }^{18}$, heißt die Parole in Uznach. «Eine Armee Penthesileas» ${ }^{19}$, die «Fühler, die in Jahrhunderten nach innen verkümmerten, steil in eiskalte Welt (streckt), (...) in die Welt auf rauhe Vorposten rückt» ${ }^{20}$, von «Mut, sogar Übermut zu den heutigen (...) Verhältnissen ${ }^{21}$ beseelt.

8 Nicht $\mathrm{zu}$ vergessen, daß es sich bei diesen schwungvollen Bekenntnissen um die Ansichten von Töchtern aus schwerreichem Hause handelt. Für jene, die über solch 
mehr als gesicherten finanziellen Hintergrund nicht verfügen, klingt das Bekenntnis zu den neuen Seinsinhalten gedämpfter. Bei 100 bis 120 DM Monatsgehalt können die Träume, wie schon bei Gilgi angedeutet, nicht sehr hoch fliegen. Ein Naturerlebnis am Wochenende, eine Zerstreuung am Oktoberfest, die Hoffnung auf eine Reise, ein neues Kleid, ein Hut, Schuhe - aber für mehr reicht es kaum im Rahmen des normalen Weiterkommens. Manchmal kommt es dann zu Ausbruchsversuchen. Die Büroangestellte Karoline z.B., die am Oktoberfest in Versuchung gerät, ihren sozialen Aufstieg durch eine Beziehung zu einem Industriellen in die Wege zu leiten: «Eine Frau, die wo etwas erreichen will, muß einen einflußreichen Mann immer bei seinem Gefühlsleben packen ${ }^{22}$, meint sie, bevor sie zum Kommerzienrat für einen Ausflug nach Altötting in den Wagen steigt. Doris (Das kunstseidene Mädchen), der es die unersprießliche Büroarbeit nie angetan hat, sieht in ihrer Entlassung die gerufene Gelegenheit, in die höhere Sphäre zu gelangen, jene Sphäre, die ihr durch den verhängnisvollen Feh-Mantel symbolisiert wird. «Ich werde ein Glanz, und was ich dann mache, ist richtig - nie mehr brauche ich mich in acht nehmen und nicht mehr meine Worte ausrechnen (...) Nichts kann mir mehr passieren an Verlust und Verachtung (...) $)^{23}$, träumt sie, und auch als sich die Zukunft nicht ganz so entwickelt, wie gewünscht, bleibt sie fest: Ohne Diplome und ohne Bildung hat sie keine Chance über die $120 \mathrm{DM}$ als Tippmamsell hinauszukommen, und sie weigert sich, in diese Tretmühle «ohne inneres Wollen und gar kein Risiko von Gewinnen und Verlieren» ${ }^{24}$ wieder einzutreten.

Wir können uns demnach nicht verhehlen, daß die soziale Dimension, die bei Sternheim durch totale Abwesenheit glänzt, wesentlich am Bild des neusachlichen jungen Mädchens beteiligt ist. Sich selbst zu verwirklichen, auf seine «persönliche Nuance» zu hören - die absolute Forderung Sternheims - scheint auf erhebliche Schwierigkeiten zu stoßen, wenn das Materielle nicht gesichert ist. Arbeiten, gerade nur um zu überleben, sich mit winzigen Freuden begnügen, wie das jung verheiratete Paar Pinneberg in Kleiner Mann, was nun? stellt sich jedoch noch als unschätzbares Privileg dar, angesichts des Damoklesschwertes des Entlassung, das auf das junge Mädchen in dem Augenblicke, wo es aufhört es zu sein - so gegen dreißig unbarmherzig niedersaust.

Dazu Siegfried Kracauer in seinem Buch Die Angestellten anhand eines Beispiels von Massenentlassungen bei «Maschinenmädchen». «Bei den lochenden Mädchen rechnet man im allgemeinen mit dem "natürlichen Abgang", d.h. man erwartet, daß sie von selber den Betrieb verlassen, wenn sie das Alter herannahen fühlen. Obwohl die Gekündigten schon über 30 Jahre zählten, wankten und wichen sie nicht (...). Man hat ihnen eine großzügige Abfindung gewährt, aber sie werden in ihrem Alter kaum wieder unterkommen (...) $)^{25}$.

11 Die Zahl der entlassenen jungen Mädchen in den von uns in Betracht gezogenen Werken ist so groß, daß man fast sagen könnte, es passiert allen! Und wenn es der vorerst am Oktoberfest übermütigen Karoline noch nicht passiert, so zum Zwecke der im Stück vorgenommenen Demonstration - es ist aber anzunehmen, daß auch für sie keine Ausnahme gemacht werden wird, und sie tut gut - vielleicht unbewußt - sich die Zuneigung des fix angestellten Zuschneiders Schürzinger zu sichern. Auch Gilgi, die Zielstrebige, wird von diesem Schicksal ereilt, so wie Christine und Fräulein Pollinger, Elisabeth und die schon genannte Doris ${ }^{26}$. 
12 Natürlich könnte man entgegnen, daß das Entlassenwerden von Angestellten - denn nur von diesen ist in den genannten Werken außer bei Sternheim die Rede - nicht nur den Frauen vorbehalten ist. Dies zu leugnen wäre absurd! Die Liste der abgebauten Männer wäre wahrscheinlich ebenso lang wie die der Mädchen, um nur Kasimir (er ist Mechaniker!), Pinneberg oder Fabian zu nennen. Die Relevanz für unser Thema entspringt nun aber daraus, zu untersuchen, warum jene Mädchen entlassen werden, und was ihnen dann als Arbeitslose zu tun übrig bleibt. Bei diesem Punkt der Untersuchung werden wir von Sternheim absehen, da sich bei ihm das junge Mädchen in einem sozial gut gepolstertem Raum bewegt.

13 Wenn sich die Zahl der arbeitenden Frauen zwischen 1907 und 1925 um $35 \%$ vergrößert hat, $\mathrm{so}^{27} \mathrm{muß}$ hinzugefügt werden, daß die Frau fast generell nur in subalternen Stellungen anzutreffen ist; in Berufen, die nur geringen Ausbildungsaufwand benötigen. «Ihre Töchter gehen in Handelsschulen, kommen als Lehrlinge in Bankbetrieben unter und werden abgebaut, wenn sie ihr volles Gehalt $\mathrm{zu}$ beanspruchen haben $»^{28}$, bemerkt lakonisch Marieluise Fleißer. Die Frau, anpassungsfähig und an Unterwerfung gewöhnt, ist «der wertvollste Aktivposten der Arbeit am laufenden Band», heißt es bei Richard Hülsenbeck, der die damals auftretende Mode der in einer Reihe tanzenden Girls als Beweis dazu anführt ${ }^{29}$.

Diese billige Arbeitskraft ohne spezielle Qualifizierung, für die die Tarifverträge nicht gelten, wird skrupellos abgebaut, vor allem dann, wenn sie auf Beförderung oder Pragmatisierung Anspruch hätte. $\mathrm{Da}$ weibliche Büroangestellte etwaigen Privatwünschen des Chefs entgegenzukommen haben, ist selbstverständlich ${ }^{30}$.

Diese Lage der Unterqualifizierung des Mädchens führt zu oft schmerzlich gewonnenen Erkenntnissen oder auch zu blindlings unternommenen Fluchtversuchen. Auf Letztere wurde schon hingewiesen - ein «Glanz» zu werden, wie Doris es metaphorisch ausdrückt, heißt Aufstieg auf mirakulöse Weise, eine Weise, die nicht auf der Basis der Arbeit beruht, aber letzten Endes auf der Ausnützung der weiblichen Reize; jener Weg, der sich für die junge Frau anno 1930 immer noch als der Sicherste erweist. Paradigmatisch dafür steht die Filmkarriere Cornelias in Kästners Fabian, die nur über den Filmbonzen und die Aufgabe des Gefährten zu erreichen ist ${ }^{31}$. Als älteres Relikt dieser fast einzigen fulguranten Aufstiegschance für die junge Frau treffen wir bei unseren Autoren die Sängerin an; wir verweisen auf die Kammersängerin Rita Marchetti in Sternheims Der Nebbich und die alternde Geliebte Herrn Koblers in Horvàths Der ewige Spießer.

16 Es ist wohl unnötig, darauf hinzuweisen, daß der Grat zwischen Aufstieg und Fall äußerst schmal ist, und die meisten dieser Träume in Halbprostitution oder Prostitution enden ${ }^{32}$. Wenn die glanzsüchtige Doris anfangs für eine goldene Armbanduhr oder drei Wochen Luxushotel mit einem Mann schläft, so muß sie es später für ein warmes Essen tun - was weit schwieriger zu bekommen ist als ein Drink!

Auch Marianne, deren Sinn eigentlich nicht so sehr nach Glanz und Luxus steht, als nach Selbstverwirklichung und Freiheit, endet zwar nicht in der Gosse - dazu hat die gutbürgerlich erzogene zu viele Skrupel - aber als Nacktplastik in einer Nachtbar. Und es ist eben Marianne, die den so legitimen Ruf des jungen Mädchens nach Bildung laut werden läßt; jene Bildung, die in der von allen Autoren außer von Sternheim dargestellten Mittelschicht den Töchtern konsequent vorenthalten wird. Wozu Geld verschwenden, ist ihre natürliche Bestimmung nicht die Ehe? «Der Papa sagt immer, die finanzielle Unabhängigkeit der Frau vom Mann ist der letzte Schritt zum 
Bolschewismus $»^{33}$. Dieses vorerst noch unreflektierte Zitat von Marianne zieht sich wie ein roter Faden durch das Stück. Im zweiten Akt doziert der Hierlinger Franz: «Eine Geliebte mit Beruf unterhöhlt auf die Dauer bekanntlich jede Liebes Verbindung, sogar die Ehe $»^{34}$, und Marianne muß diesen männlichen Versklavungsgrundsatz im Laufe des Stückes teuer mit ihrem Lebensglück bezahlen. Nach dem Tod ihrer Mutter vom Vater zugleich als Dienstbote im Haus und als Verkäuferin im Scherzartikelgeschäft eingesetzt, - und weil man diese billige Kraft doch nicht ständig behalten kann - im heiratsfähigen Alter an die solide Fleischhauerei Oskars verkuppelt, glaubt Marianne im letzten Moment ausbrechen zu können. Da sie aber keinerlei Berufsausbildung besitzt, kann ihr «Retter» mur in Form eines männlichen wesens erscheinen. Blind und taub für Alfreds berufliche und menschliche Unzulänglichkeit wirft sie sich ihm an den Hals, bricht die Verlobung mit Oskar und glaubt sich durch die Liebe gerettet.

Alfred ist jedoch keineswegs gewillt noch fähig, für Frau und Kind zu sorgen und entledigt sich seiner Gefährtin indem er sie aktiv in das Nachtleben Wiens einführt. Er kann nun Distanz nehmen, und der Ausspruch von der «berufstätigen Frau, die das Leben des Paares unterhöhlt», ist auf zynische Weise bewahrheitet.

Als der Vater entrüstet seine verstoßene Tochter in ihrer nächtlichen Tätigkeit entdeckt, schleudert sie ihm ins Gesicht: «Nein, das kann ich mir nicht leisten, daß mich schäm'». Ich verdien' zwei Schilling pro Tag. Das ist nicht viel, inklusive den kleinen Leopold. Was kann ich denn aber auch anderes unternehmen? Du hast mich ja nichts lernen lassen, du hast mich ja nur für die Ehe erzogen $»^{35}$.

Mariannes Schicksal verweist uns direkt auf Frieda Geyer, die, zwar nicht speziell dafür ausgebildet, mit eiserner Härte versucht, sich in ihrem Vertreterberuf durchzusetzen. Sie ist nicht bereit, ihre schwer errungene Selbständigkeit den Machtansprüchen eines zukünftigen Gatten zu opfern. Gustl Amricht, Schwimmkanone und angehender Kaufmann, fordert stracks von ihr die Aufgabe des Berufs, die Mitarbeit im Geschäft und hat sogar ein Auge auf das Erbteil der kleinen Schwester Friedas geworfen. «Das Recht auf Deine Arbeitskraft steht sowieso deinem Mann zu» ${ }^{36}$, behauptet Gustl, um etwas später angesichts der hartnäckigen Weigerung Friedas die Drohung auszustoßen: «Du wirst mir nicht entgehen»! ${ }^{37}$ Das sind genau dieselben Worte, die der verschmähte Fleischermeister Oskar der ihm entschlüpften Marianne nachruft! Bei beiden Männern schlägt die Liebe augenblicklich in grausamen $\mathrm{Ha}$ um: «Du wirst mich heiraten oder ich werde dich zurichten, daß niemand mehr etwas mit dir zu tun haben will» ${ }^{38}$, droht Gustl; und Oskar schwelgt in biblischen Vergeltungsvisionen: «Gottes Mühlen mahlen langsam, mahlen aber furchtbar klein (...) wen Gott liebt, den prüft er. - Den straft er. Den züchtigt er. Auf glühendem Rost, in kochendem Blei (...)» $)^{39}$.

21 Frieda entkommt mit knapper Not der von ihrem Verschmähten angezettelten Lynchjustiz - um welchem fraglichen und einsamen Schicksal entegegenzugehen? Marianne muß sich geschla-gen und verstört in das Prokrustesbett der Ehe mit Oskar bequemen, der für seine Rache an ihr ein ganzes Leben vor sich hat. «Schau, die Mariann'», das ist doch nur ein dummes Weiberl (...), und die hat sich eigebildet, die Welt nach ihrem Bild umzuformen (...) $»^{40}$, bemerkt dazu die angepaßte Mittfünfzigerin Valerie, deren Selbständigkeit durch eine Rente und eine Tabaktrafik gesichert ist.

Welches sind nun die Möglichkeiten für das junge Mädchen, das sich zwischen den zwei Kriegen mutig in die Welt gestürzt hat, um sein Glück als eigenständiges Individuum zu erproben? Es will uns scheinen, daß die Antwort recht bescheiden lautet. Für Mädchen aus einfachen Verhältnissen, die einen Beruf ergreifen wollen, sind die Chancen schon 
dadurch gering, daß ihre Familien zu keinem Opfer für ihre Ausbildung bereit sind. In subalternen Stellungen ohne Aufstiegsmöglichkeit heißt es dann, sich mit dem Wenigsten begnügen, und nur die Ehe steht als halbwegs realistischer Ausweg offen, um der Arbeitslosigkeit nach dreißig $\mathrm{zu}$ entgehen. Auf grundlosen Illusionen beruhende Ausbruchsversuche - siehe Doris, Karoline, Marianne - enden kläglich bis gefährlich - der Traum von Film bleibt ein Traum, außer bei Kästner, der in seinem misogynen Buch die Anpassungsfähigkeit der Frau der geraden Aufrichtigkeit des Mannes gegenüberstellen wollte. Prostitution, wie bei Horvàths Anna oder Agnes ${ }^{41}$, oder quasi Prostitution wie bei Doris, sind sehr oft das Ende beim Traum von der Selbständigkeit.

Die Mädchen, die auf ihrer Selbständigkeit bestehen und sich weder versklaven wollen, noch den Partner mit einer Dauerbindung belasten wollen, wie z.B. Frieda Geyer oder Gilgi - sie verläßt den finanziell zu knapp gestellten geliebten Mann, von dem sie ein Kind erwartet, um ihn in seinen Lebensgewohnheiten eines Privatiers nicht zu stören jene Mädchen steuern einem ungewissen, schweren Schicksal entgegen. Wird es Gilgi trotz Arbeitswillens gelingen, ihr Kind alleine durchzubringen? Wird sich Frieda auf die Dauer erfolgreich gegen die Männerkonkurrenz durchsetzen können? Oder wird auch sie wie Elisabeth in Horvàths Glaube, Liebe, Hoffnung, die durch unverdiente Schwierigkeiten mit ihrem Wandergewerbeschein mit dem Gesetz in Konflikt gerät, absinken und sogar mit Selbstmord enden?

Die selbständigen Flugversuche unseres neuen jungen Mädchens scheinen also kaum einen brillanten Ausgang $\mathrm{zu}$ nehmen. Widrige Zeitumstände, wirtschaftliche und politische Stürme erschweren das Unternehmen, und die meisten landen irgendwo, physisch und psychisch angeschlagen, oder zerstört. Wenn auch die wirtschaftliche Krise bei diesem Mißerfolg nicht $\mathrm{zu}$ unterschätzen ist, so ist sie nicht alleine verantwortlich zu machen, und bei der Vereitelung der Selbstentfaltung des jungen Mädchens scheint uns ein nicht unwesentlicher Faktor ihr Partner selbst zu sein mit der von ihm etablierten patriarchalischen Moral. «'Moral' das ist der Titel für die Disziplinierung der Frau, die sich "der ökonomischen Verwertung" verweigert $»^{42}$, formuliert Lethen im Hinblick auf Fleißers Roman; und Alfred, das Horvàthsche Paradebeispiel des männlichen Durchschnitts behauptet kalt: «Eine rein menschliche Beziehung wird erst dann echt, wenn man was voneinander hat» ${ }^{43}$.

Alfred Klostermeyer, Schupo und Rettungsanker der gescheiterten und zur Ehe resignierten Elisabeth ${ }^{44}$, ergeht sich in Selbstmitleid, als die Polizei seine Braut stantepede als «öffentliche Ruhestörerin» arrestiert, weil sie keinen fixen Wohnort aufweisen kann. «Polizeiwidrig ist nicht, wer kein Unterkommen hat, polizeiwidrig ist, wer dadurch die öffentliche Ordnung gefährdet» ${ }^{45}$, kommentiert die Sittenpolizei, und Alfons findet an der Leiche Elisabeths keine anderen Worte als «ich hab kein Glück, ich hab kein Glück» ${ }^{46}$.

Glück haben wollen - ein legitimes Verlangen des Menschen, ob Mann oder Frau - aber daß die Frau diesem Bedürfnis außerhalb der männlichen Einflußsphäre nachgeht, scheint wenigen männlichen Exemplaren der in Betracht gezogenen Werke vertretbar zu sein. Ob Lustobjekt oder Zweckobjekt, oder beides - der Glücksanspruch des Mannes richtet sich wesentlich an die Frau.

Angesichts der Lockerung der Moral in den Nachkriegs jähren ist das junge Mädchen oft in erster Linie Lustobjekt. Neu dabei ist, daß auch die Mädchen Anspruch auf Lusterfüllung erheben: «Die Welt hat sich gedreht, mein Herr, und warum sollen nur 
die Männer Don Juane sein dürfen? $\gg^{47}$, heißt es in Horvàths Stück Don Juan kommt aus dem Krieg. In diesem Sinne dringen bei Sternheims Schule von Uznach Sonja und Maud nächtlicherweise in Klaus' Zimmer um ihn mit ihrer Gunst zu bestürmen. In diesem Falle bleibt das Fiasko psychologisch - der junge Herr verschmäht groben Sinnengenuß und verweist die jungen Damen auf ihre Plätze. Bei den sozial tiefer ansetzenden Autoren wird die Aufforderung, bzw. Bereitschaft meist nicht zurückgewiesen, aber die daraus entstehenden Schwierigkeiten werden dann durchaus der Frau überlassen. Christine in Horvàths Stück Zur schönen Aussicht, Agnes Pollinger, Marianne, Gilgi, und nicht $\mathrm{zu}$ vergessen Lämmchen - obzwar verheiratet - müssen salbungsvollen, aber unbeugsamen Ärzten gegenüber ihre ungewünschte Mutterschaft - meist alleine - auf sich nehmen. «Ihr wollt den Warencharakter der Liebe, aber die Ware soll verliebt sein. Ihr zu allem berechtigt und zu nichts verpflichtet, wir zu allem verpflichtet, und $\mathrm{zu}$ nichts berechtigt $»^{48}$, analysiert die Akademikerin Cornelia.

Besonders kraß wird diese Behauptung in Zur schönen Aussieht illustriert: Christine, durch Straßer, einen brutalen Tunichtgut Mutter geworden, bewahrt ihm ihre Liebe und schreibt ihm beharrlich. Als sie auf sein Schweigen hin nach langen Monaten selbst auftaucht, verbrüdern sich die anwesenden Männer zu einer infamen Komödie, um Straßer vor der Vaterschaft zu bewahren: Jeder soll vorgeben, mit Christine ein Verhältnis gehabt zu haben. Als die Mystifikation im besten Schwünge ist, stellt sich heraus, daß Christine 10.000 DM geerbt hat!

Horvàth nennt diese finanziellen Rettungen «den lieben Gott». «Ich wurde abgebaut, und wenn der liebe Gott mir nicht geholfen hätte, wäre ich untergegangen ${ }^{49}$, bekennt Christine. Was wunders, wenn die Zuneigung der Partner plötzlich ins Inkommensurable wächst! «Ich hab' ja schon immer von der Erlösung durch das Weib geträumt, aber ich hab's halt nicht glauben können $»^{50}$, gesteht Karl in der Italienischen Nacht, als Leni ihm eröffnet, sie besitze $4.000 \mathrm{DM}$, die sie ihm zur gemeinsamen Einrichtung eines Kolonialwarenladens anträgt. Hier haben wir den genauen Gegensatz zu Frieda, der Ehrgeizigen, aber wir dürfen nicht vergessen, daß der Autor in einem Fall ein Mann, im anderen eine Frau ist.

Es stimmt, daß Horvàth zwischen 1926 und 1933 seinen Fräulein-Figuren großes Mitleid entgegenbringt, aber es ist zu bedenken, daß es sich meist um recht anspruchslose Durchschnittswesen handelt, die fast automatisch unter die Räder der patriarchalischen Gesellschaft kommen ${ }^{51}$. Dahinter scheint aber schon auch bei Horvàth eine geheime Sehnsucht nach den paradiesischen Zuständen des Verstehens und Eingehens zu schwelen, meist im Negativ dargestellt, manchmal angedeutet, wie z.B. zwischen Kasimir und Karoline, bzw. Kasimir und Erna. «Solange wir uns nicht aufhängen, werden wir nicht verhungern $\aleph^{52}$, heißt Ernas trostreicher Minimalanspruch. Die Selbstaufgabe im Paar hin bis zu den schlimmsten Verhältnissen ist ja auch Lämmchens unerschütterliche Haltung - oder besser gesagt, die von Fallada an die Frau herangetragene Erwartung.

Wenn 1936 in Die Unbekannte aus der Seine Ernst zur Überzeugung kommt: «Den wahren Frieden gibt uns nur die Frau, denn das Weib repräsentiert die Natur» ${ }^{53}$, so ergibt die Ökonomie des Stückes hier einen noch ironischen Sinn; wenn aber kurz darauf die reuige Irene erkennt: «Ich hab (...) gelesen, daß die Frau die Pflicht hat, die Härte der Paragraphen durch die Liebe zu erweichen, aber jetzt ist es wohl zu spät» ${ }^{54}$, so ist es genau das, was die Unbekannte mit ihrem Opfertod demonstriert, und Irene so die Gelegenheit verschafft, ihr Versäumnis nachzuholen. Und wenn gar die Gräfin in Figaro 
läßt sich scheiden (1936) an Susanna schreibt: «Eine Frau gehört zu ihrem Mann» ${ }^{55}$, so muß die Aussage wortwörtlich genommen werden, steckt denn nicht in dem Stück ein Plädoyer für Familie und Kindersegen, ungeachtet der herrschenden Zeitumstände? Fanchette behauptet rundheraus: «Eine Frau ohne Kind ist gar keine Frau» ${ }^{56}$ Überzeugung, an der Susanne angesichts des störrigen Figaro schon lange leidet.

Die Tatsache, daß sich bei Horvàth im Hinblick auf die Einschätzung der weiblichen Entfaltungsmöglichkeiten ein bedeutender Umschwung ereignet hat, erweist sich erst bei genauer und chronologischer Lektüre seines Werkes; bei Sternheim hingegen, unserem zweiten Bezugspunkt, liegen die Dinge viel einfacher.

Das von ihm so frenetisch begrüßte junge Mädchen: «Hätte der Krieg nur die Nummer, das neue Mädchen hervorgebracht, viel ist von seinem Grauen ausgelöscht» ${ }^{57}$ - wird praktisch schon im selben Atemzug verurteilt. Dieser Schrei der Begeisterung des Siebensternsohnes aus dem 2. Akt wirkt suspekt vor dem Hintergrund seiner Äußerungen am Ende des 1. Aktes: «Das Mädchen aus seiner schäbigen Mitgenommenheit und Abgegriffenheit verdient großes Mitleid» ${ }^{58}$; und kruz darauf, in liebliche Träumerei versunken, ist ihm der gymnastische Elan seiner weiblichen Hausund Zeitgenossen schon «klotziger Impetus, (...) seelisches Erbeben und Erbrechen»" Empört über den nächtlichen Überfall zweier liebeshungriger Pensionärinnen schleudert er dann der neu angekommenen Mathilde Enterlein ohne Vorbehalt den Grund seiner männlichen Seele ins Gesicht: «Mag denn des Mädchens Gelüst, das Leben zu fressen, berechtigt sein. In Euren Manieren, dem plumpen Auftritt (...) wirkt Ihr grauenhaft; fähig, das von uns erträglich gestaltete Bild des Weibes zur Fratze, vor der wir uns verhüllen, zu entstellen. Wir, Eure Partner im ernsten Spiel, müssen Euch jetzt vor schlimmsten Entgleisungen warnen (...), daß ein Mann ein Schurke sein kann, ist für das repräsentative Frauenzimmer kein Grund, ihn nachzuahmen (...). Was vermochte Sie, um Gottes Willen, sich so zu verramschen, jedem Kommis (...) um den ungewaschenen Hals zu fallen? (...) Wollen Sie die männliche Seele, die auf Erden nur noch über des Mädchens makellose Reinheit zu jauchzen hat, tödlich verletzen ${ }^{60}$ ?

Klaus' Empörung Mathilde gegenüber ist falsch am Platze - sie stammt aus Lüneburg ${ }^{61}$ und ist noch Jungfrau! Klaus' männliche Seele kann in ihr das «lotende Senkblei» ${ }^{62}$ finden - aus ihnen wird ein Paar, das alle Prinzipien Uznachs über den Haufen wirft und zu reihenweisen Findungen und Hochzeiten führt. Die «Exzesse der Affektation erzwangen (...) den Gegenstoß empörter Natur» ${ }^{63}$, kommentiert Siebenstern, und damit bedarf es wohl keiner weiteren Worte mehr, um zu beweisen, daß Sternheim zwar vom neusachlichen jungen Mädchen beeindruckt, aber in erster Linie beängstigt ist; daß sein Wortschwall zu dessen Darstellung mehr einer magischen Beschwörungsformel des Übels, als einem Preislied gleicht.

Es mag sich bilden, es mag auf der Höhe des Zeitgeistes stehen - die absolute und unabdingbare Bestimmung des jungen Mädchens ist die Hingabe an den Mann, einen Mann, der von Sternheim natürlich ebenso exemplarisch gedacht ist wie das unverbildete Prachtmädchen Mathilde.

Die Sternheimschen Exempel an Virilität brauche ich nicht weiter vorzustellen, sie sind seit der Komödienreihe Aus dem bürgerlichen Heldenleben wohl bekannt: selbstischer, ja selbstbesessen, jenseits von Gut und Böse, und, nicht $\mathrm{zu}$ vergessen, mit außerordentlicher Potenz begabt, «pure, klotzige Männlichkeit»" ${ }^{64}$, wie dies die verwöhnte Kammersängerin Rita bezüglich ihres Partners Fritz Tritz formuliert. «Ich dampfe, rausche bei dir mit der ganzen Haut, bin in allen Nerven gesprengt ${ }^{65}$. 
Es ist anzunehmen, daß dieses strahlende Schicksal auch Klara Cassati zuteil werden wird, wenn sie erst den Panzer ihrer "gefrorene) Vernunft» ${ }^{66}$ gesprengt hat, dann wird auch sie, «anden Brüsten sprühen $»^{67}$, so wie die neue Partnerin Rene Maria Blands, der, des Intellekts der überfeinerten Yvette müde, die Freuden des «wahren Weibes» erlebt. «Nichts kommt darauf an, ob sie lacht oder weint, vom Sinn ihrer Worte, von ihrer Entschlüsse Wert hängt nicht das Geringste $a b$ (...), stets entsteigt ihr das Ursprüngliche, von dem Gebären und Frucht (...) kommt» ${ }^{68}$.

Bei genauem Hinsehen können wir uns also nicht verhehlen, daß Sternheim hinter den ambivalenten Visionen vom neusachlichen jungen Mädchen der Frau im Grunde jegliche Bedeutung abspricht, die nicht die des totalen Aufgehens im männlichen Partner wäre. Als die hingebungsbereite Evelyne in Aut Caesar, aut nihil ihren angebeteten Dichter fragt, was die junge Frau tun soll, so antwortet dieser: «Die junge Frau soll lieben wie du, damit Liebe auf Erden kein Märchen wird» ${ }^{69}$. Da die soziale Dimension bei Sternheim nicht vorhanden ist, so darf in dieser Selbstaufgabe aber kein Opfer gesehen werden, so wie z.B. Lämmchen es bringt, indem sie mit niedrigen Arbeiten die Familie vor dem Hunger bewahrt; es ist im Gegenteil bei Sternheim klar, daß Selbstaufgabe der Frau mit Selbstverwirklichung geichgesetzt wird, da die männliche Potenz den weiblichen Partner mehr als entschädigt, und es ist wohl nicht übertrieben $\mathrm{zu}$ behaupten, daß Sternheims Ansätze über das neusachliche junge Mädchen in einer vitalistischen Orgie enden, wo gesteigerter Geschlechtsakt und Fortpflanzung apotheotisch verklärt sind. Die Skizze Die Väter oder knock out endet mit dem Bild der jungen Mutter Fantasia, die an ihren Brüsten die beiden im Irrenhaus internierten Dichter, Bromme und Bendorf säugt, und Aut Caesar, aut nihil endet mit dem triumphalen Schrei «doch bin ich in der Zeit ein Riese: Aut nihil, aut Caesar» ${ }^{70}$, nachdem Caesar Kinkel erfahren hat, daß drei Berliner Freudenmädchen ihn fast zugleich zum Vater gemacht haben -noch dazu von Zwillingen!

Wenn man nun das Fazit unserer Betrachtungen ziehen will, so könnten wir etwas polemisch überspitzt fragen, ob es nicht gerade Sternheim, der von der Realität am weitesten entfernte ist, der die repräsentativste Aussage zum Thema macht? Sternheim sagt, was alle männlichen Autoren irgendwie träumen oder nicht zu sagen wagen: die Frau gehört zum Manne und ihr bestes Geschäft ist immer noch Kinderkriegen, auch dann, wenn die Umstände nicht gerade vorteilhaft sind. Wenn auch von einigen die Anstrengungen des jungen Mädchens mit Sympathie betrachtet werden, so steckt doch immer geheime Angst dahinter, die beim ersten Anlaß in offene Forderung oder Anklage umschlägt. Anders bei den beiden weiblichen Autoren: Nachdem das neusachliche junge Mädchen zum Bewußt stein seiner selbst gelangt ist, ist der Traum vom schützenden und helfenden Mann radikal ausgeträumt. Die Tatsache, daß sich der Mann immer als Feind einer nach Selbstbehauptung strebenden Frau darstellt, stößt hart auf die Tatsache des aufeinander Angewiesenseins der beiden Geschlechter. Alle drei Romane enden in Dissonanz. Ist das eine Zeiterscheinung der neuen Sachlichkeit, oder soll sich diese Dissonanz einmal in Harmonie auflösen? Es steht zu befürchten, daß sich auch in den sechzig Jahren, die inzwischen verstrichen sind, nichts Wessentliches daran geändert hat. 


\section{NOTES}

2. - Livia Z. Wittmann: Der Stein des Anstoßes, Zu einem Problemkomplex in berühmten und gerühmten Romanen der Neuen Sachlichkeit, Christchurch, New Zealand, Jb. für internat. Germanistik, Berlin, Frankfurt, 14/1982/2, S. 83.

3. - Alle diese jungen Frauen mit großem Vermögen sind mit großer Wahrscheinlichkeit als Spiegelung seiner Frau Thea Bauer anzusehen.

4. - Carl Sternheim, Gesamtwerk hsg. von Wilhelm Emrich, Luchterhand Verlag 1963, Bd. 3 S. 21.

5. - Ebda S. 31.

6. - Ebda S. 26.

7. - Ebda S. 27.

8. - Marieluise Fleißer, Eine Zierde für den Verein (ursprünglicher Titel, Mehlreisende Frieda Geyer, 1931), Suhrkamp taschenbuch 294, Frankfurt, 1972, S. 36.

9. - Entstanden 1925/26.

10. - Uraufführung $18 / 11 / 1932$.

11. - Carl Sternheim: 1878-1942, Ödön v. Horvàth: 1902-1938.

12. - Mehlreisende Frieda Geyer, zitiert nach Helmut Lehten, Neue Sachlichkeit, Studien zur Literatur des Weißen Sozialismus, Metzler, Stuttgart, 1979, S. 32.

13. - Irmgard Keun, Gilgi, eine von uns, Düsseldorf, 1979, S. 70.

14. - Gesamtwerk, Bd. 3, S. 382.

15. - Ebda, S. 380.

16. - Ebda.

17. - Ebda, S. 379.

18. - Ebda, 403.

19. - Ebda, S. 380.

20. - Ebda.

21. - Ebda, 379.

22. - Ges. Werke Bd. 1, S. 279.

23. - Irmgard Keun, Das kunstseidene Mädchen, Düsseldorf, 1979, S. 45.

24. - Ebda, S. 181.

25. - Zitiert nach Dieter Mayer, Hsg: Kleiner Mann, was nun? Frankfurt, Berlin, München, 1978, S. 37.

26. - Der Reihe nach aus: Zur schönen Aussicht, Der ewige Spießer, Das Märchen vom Fräulein Pollinger, Glaube, Liebe, Hoffnung (Horvàth), und Das Kunstseidene Mädchen.

27. - Dieter Mayer, Ebda, S. 34.

28. - M. Fleißer, Ebda, S. 108.

29. - Helmut Lethen, Ebda, S. 36.

30. - Gilgi hilft sich aus der Affäre, indem sie den Chef ihrer gewitzten Freundin Olga überläßt, und Doris, die sich weigert, wird stracks entlassen.

31. - Erich Kästner, Fabian, Ges. Schriften, Bd. 2, Köln, Berlin, O.J.S. 129.

32. - Dazu Siegfried Kracauer in Die Angestellten, zitiert nach D. Mayer, Ebda, S. 39: «Nichts kennzeichnet so sehr dieses Leben, (...) als die Art und Weise, in der ihm das höhere erscheint. Es ist ihm nicht Gehalt, sondern Glanz. Es ergibt sich ihm nicht durch Sammlung, sondern durch Zerstreuung.

33. - Ö.v. Horvàth, Geschichten aus dem Wienerwald, Ges. Werke, Bd. 1, S. 177.

34. - Ebda, S. 201. Siehe auch Zur schönen Aussicht, Ebda, Bd. 3, S. 40: «Berufstätige Fräulein unterhöhlen das bürgerliche Familienleben».

35. - Ebda, Bd. 1, S. 232. 
36. - M. Fleißer, Edba, S. 127.

37. - Ebda, S. 140.

38. - Ebda, S. 144.

39. - Ö.v. Horvàth, Ebda, Bd. 1. S. 214.

40. - Ebda, S. 242.

41. - Der ewige Spießer und Das Märchen vom Frl. Pollinger.

42. - H. Lethen, Ebda, S. 173.

43. - Horvàth Ebda, Bd. 1, S. 163.

44. - Ebda, S. 349, Elisabeth: «Ich hab' immer selbständig sein wollen so mein eigener Herr. Maria: Das geht nicht».

45. - Ebda, S. 359.

46. - Ebda, S. 379.

47. - Ebda, Bd. 2, S. 610.

48. - E. Kästner Ebda, S. 74.

49. - Horvàth Ebda, Bd. 2, S. 26.

50. - Ebda, Bd., 1. S, 144.

51. - Dazu eine Ausnahme, Anna, die Politische in Die Italienische Nacht.

52. - Ebda, S. 323.

53. - Ebda, Bd. 3 S, 157.

54. - Ebda, S. 190.

55. - Ebda, Bd. 4, S. 435.

56. - Ebda, S. 444.

57. - C. Sternheim, Ebda, Bd. 3, S. 414.

58. - Ebda, 392.

59. - Ebda, S. 421.

60. - Ebda, S. 429.

61. - Auch Lili von Hessenkamp in Sternheims Spätdrama John Pierpont Morgan stammt aus der unberührten Provinz (Oldenburg) und ist von erquickender Frische.

62. - Ebda, S. 430.

63. - Ebda, 440.

64. - Ebda, S. 221.

65. - Ebda, S. 204.

66. - Ebda, S. 21.

67. - Ebda, Bd. 4, S. 312.

68. - Ebda, S, 312.

69. - Ebda, Bd, 10/1, S. 112.

70. - Ebda, S. 119.

1. - Ödön von Horvàth: Gesammelte Werke, Frankfurt/Main, 1972, Bd. 3S.70.

\section{RÉSUMÉS}

Gibt es ein «neusachliches» junges Mädchen? Carl Sternheims Stück, Die Schule von Uznach oder die neue Objektivität scheint diese Frage direkt aufs Korn zu nehmen. Aber auch Ödön von Horvàth, Erich Kästner, Hans Fallada, Irmgard Keun und Marieluise Fleißer haben sich in ihren Werken 
mit dem «Phänomen» eingehend beschäftigt. Die Antwort ist eindeutig und einstimmig: Ja, das junge Mädchen der Zwischenkriegszeit hat sich geändert; es ist ambitioniert, weltoffen, selbständig, sportlich und unsentimental. Ob dieses junge Mädchen aber auf dem richtigen Weg ist, und ob es diesen Weg wird verwirklichen können - hier lauten die Antworten sehr verschiedentlich, verschieden vor allem, je nachdem, ob es sich um einen Autor weiblichen oder männlichen Geschlechts handelt...

La jeune fille de la Nouvelle Objectivité existe-t-elle? Carl Sternheim, dans sa pièce L'École d'Uznach ou la Nouvelle Objectivité semble directement aborder cette question. Or, Ödön v. Horvàth, Erich Kästner, Hans Fallada, Irmgard Keun et Marieluise Fleißer ont également cerné ce "phénomène » dans leurs œuvres - et leur réponse à tous est unanime : Oui, la jeune fille de l'entredeux-guerres a changé, elle est ambitieuse, ouverte, indépendante, sportive et objective. Toutefois, à la question de savoir si cette jeune fille est sur le bon chemin, si elle pourra aller jusqu'au bout sur cette voie, les réponses divergent, elles divergent notamment selon le sexe de l'auteur: les hommes ne répondent pas de la même façon que les femmes...

\section{AUTEUR}

\section{EVA PHILIPPOFF}

Université de Limoges 